\title{
Hot magnetized nuclear matter: Thermodynamic and Saturation Properties
}

\author{
Z. Rezaei $^{1} *$, G. H. Bordbar ${ }^{1,2}$ \\ ${ }^{1}$ Department of Physics and Biruni Observatory, \\ Shiraz University, Shiraz 71454, Iran $^{\dagger}$, \\ and \\ ${ }^{2}$ Research Institute for Astronomy and Astrophysics of Maragha, \\ P.O. Box 55134-441, \\ Maragha 55177-36698, Iran
}

\begin{abstract}
We have used a realistic nuclear potential, $A V_{18}$, and a many body technique, the lowest order constraint variational (LOCV) approach, to calculate the properties of hot magnetized nuclear matter. By investigating the free energy, spin polarization parameter, and symmetry energy, we have studied the temperature and magnetic field dependence of the saturation properties of magnetized nuclear matter. In addition, we have calculated the equation of state of magnetized nuclear matter at different temperatures and magnetic fields. It was found that the flashing temperature of nuclear matter decreases by increasing the magnetic field. In addition, we have studied the effect of the magnetic field on liquid gas phase transition of nuclear matter. The liquid gas coexistence curves, the order parameter of the liquid gas phase transition, and the properties of critical point at different magnetic fields have been calculated.
\end{abstract}

\footnotetext{
* Corresponding author. E-mail: zrezaei@shirazu.ac.ir

$\dagger$ Permanent address
} 


\section{INTRODUCTION}

Dense nuclear matter at high temperatures and strong magnetic fields can be found in the formation of protoneutron stars and relativistic heavy ion collisions. In such systems, the temperature reaches $20-50 \mathrm{MeV}$ [1, 2] as well as magnetic fields up to $10^{19} \mathrm{G}$ [3]. Therefore, investigation of the hot and magnetized nuclear matter is one of the important problems in both astrophysics and nuclear physics.

Many authors have studied the properties of nuclear matter at high temperatures [4-9]. Applying a finite temperature nuclear Thomas-Fermi model with a Wigner-Seitz approximation, the clustering of nucleons in hot dense matter has been studied [4]. It has been concluded that the thermodynamic variables are fairly insensitive to the exact arrangement of the nucleons, while the sizes of the nuclear clusters exhibit an uncomfortable sensitivity to relatively small effects. A fully self-consistent model with an effective interaction has been used to derive the temperature dependence of different thermodynamic quantities of nuclear matter [5]. It has been found that the calculated entropy is in agreement with the experiment. The variational method and realistic nuclear potentials have also been applied to construct the equation of state for nuclear matter at finite temperatures [6]. Within the static fluctuation approximation and using the Reid68 and Reid93 soft-core potentials, the bulk and thermodynamic properties of nonrelativistic hot nuclear matter have been considered [7]. A variational theory for fermions at finite temperature and high density has been applied to study the equation of state of symmetric nuclear matter and the behavior of the nucleon effective mass in medium, and the fate of the neutral pion condensation at finite temperature [8]. The equation of state of hot polarized nuclear matter has been applied to simulate the repulsive force caused by the incompressibility effects of nuclear matter in the fusion reactions of heavy colliding ions [9]. The results show that the temperature effects of compound nuclei have significant importance in simulating the repulsive force on the fusion reactions.

One of the important quantity in nuclear matter to study the ferromagnetic phase transition, the equation of state and the structure of neutron rich nuclei and protoneutron stars is the symmetry energy of nuclear matter. It has been concluded that there is a correlation of the crust-core transition density and pressure in neutron stars with the slope of the symmetry energy and the neutron skin thickness [10]. In addition, the sensitivity of 
nuclear symmetry energy elements at the saturation density to the binding energies of ultra neutron-rich nuclei and the maximum mass of neutron star has been also considered [11]. It has been shown that a strong correlation of the neutron star radii with the linear combination of the slopes of the nuclear matter incompressibility and the symmetry energy coefficients at the saturation density can be exist [12]. Since the nuclear matter can be at high temperatures, it is necessary to investigate the symmetry energy of hot nuclear matter. Applying a consistent Hartree-Fock study of the equation of state (EOS) of asymmetric nuclear matter at finite temperature, it has been confirmed that there is a strong impact of the symmetry energy and nucleon effective mass on thermal properties and composition of hot protoneutron star matter [13]. In the framework of the local density approximation, the temperature dependence of the symmetry energy for isotopic chains of even-even $\mathrm{Ni}, \mathrm{Sn}$, and $\mathrm{Pb}$ nuclei has been explored [14]. The results show that the symmetry energy decreases with temperature.

Studying the liquid gas phase transition of hot nuclear matter has also been considered in some researches [15 28]. According to the data from the heavy ion collisions [15] and nuclear reaction experiments [16], the critical temperature of the liquid gas phase transition is $16.6 \mathrm{MeV}$ and $17.9 \mathrm{MeV}$, respectively. This is while the models and interactions affect the critical temperature obtained using the theoretical calculations. The results are $17.5 \mathrm{MeV}$ [17], 13.6-18.3 MeV [18], $21 \mathrm{MeV}$ and $20 \mathrm{MeV}$ [19], 16.2 MeV [20], $15-20 \mathrm{MeV}$ [21], $13 \mathrm{MeV}$ [22], $14-23 \mathrm{MeV}$ [23], $14.7 \mathrm{MeV}$ [24], and $15.7 \mathrm{MeV}$ [25] using different models. Within the Self-Consistent Greens Functions approach and employing $A V_{18}$ potential, the results for the critical and flashing temperatures are $11.6 \mathrm{MeV}$ and $9.5 \mathrm{MeV}$ [26]. Applying microscopic nuclear forces from the chiral effective field theory, the critical temperature has been determined to be between 17.2 $\mathrm{MeV}$ and 19.1 $\mathrm{MeV}$ [27]. The thermal properties and the liquid gas phase transition of symmetric nuclear matter have been also investigated using a standard covariance analysis [28]. The results for the critical temperature lie between $14 \mathrm{MeV}$ and $18 \mathrm{MeV}$. Besides, the flashing temperature is within $11 \mathrm{MeV}$ and $13 \mathrm{MeV}$.

Strong magnetic fields created in dense nuclear media also affect the thermodynamic properties of such systems. Different studies have explored the nuclear matter in the presence of strong magnetic fields [29 34]. In a relativistic Hartree theory, it has been indicated that the application of magnetic field leads to additional binding for the system with a softer equation of state [29]. Besides, the saturation density of nuclear matter increases by 
increasing the magnetic field. Using the relativistic nuclear models, it has been found that the presence of the magnetic field will increase the instability region [30]. Effective baryonmeson exchange models with magnetic field coupled to the charge and the dipole moment of the baryons have also confirmed that by increasing the magnetic field, the saturation density of nuclear matter increases and the system becomes less bound [31]. In addition, it has been shown that as the magnetic field increases, the system becomes more incompressible. In the framework of the relativistic mean field models FSU-Gold, it has been confirmed that at low densities and by increasing the magnetic field, the energy per particle turns out to be increasing lower and a softening of the equation of state appears [32]. However, at high densities, while the softening of the equation of state (EOS) will be gradually overwhelmed by stiffening resulting from the anomalous magnetic moments effect, the energies are slightly reduced by a strong magnetic field. Relativistic field-theoretical models for nuclear matter have been applied to study the creation of nuclear matter in a sufficiently strong magnetic field [33]. It has also been clarified that nuclear matter is more strongly bound in a magnetic field. The equation of state, the compressibility, and magnetic susceptibility of nuclear matter in the presence of a magnetic field have been investigated employing the non-relativistic Skyrme potential model within a Hartree-Fock approach [34].

One of the many-body approaches in nuclear systems is the lowest order constraint variational (LOCV) method [35-37]. This approach has been extended to the finite temperature for neutron matter, nuclear matter, and asymmetrical nuclear matter [38 41]. This method is fully self-consistent, with no free parameters into the calculations. In addition, this approach applies a normalization constraint [43] leading to small values for the higher-order terms [37, 41, 42]. Besides, to perform an exact functional minimization of the two-body energy with respect to the short-range behavior of the correlation function, a particular form for the long-range behavior of the correlation function is assumed. Therefore, we obtain a computational simplification over the unconstrained methods to parameterize the shortrange behavior of the correlation functions. In our previous studies, we have investigated the polarized nuclear matter at finite temperature applying LOCV method using realistic nuclear potentials in the absence of the magnetic field [44 49]. In addition, we have recently studied the properties of magnetized nuclear matter at zero temperature [50, 51]. In this paper, we are interested in the saturation and thermodynamic properties of magnetized nuclear matter at finite temperatures using LOCV method applying $A V_{18}$ potential. 


\section{LOCV CALCULATIONS FOR MAGNETIZED NUCLEAR MATTER AT FI- NITE TEMPERATURE}

In this work, we propose a system of pure homogeneous symmetric nuclear matter at finite temperature in the presence of a uniform magnetic field $B$ along the $z$-axis. Our system contains $A$ nucleons with spin-up (+) and spin-down (-). The number densities of nucleons with the isospin and spin projection $j$ and $i$, respectively, are denoted by $\rho_{j}^{(i)}$. In addition, $\rho^{(i)}=\rho_{p}^{(i)}+\rho_{n}^{(i)}$ presents the number density of nucleons with the spin projection $i$. We introduce the spin polarization parameter by $\delta=\left(\rho^{(+)}-\rho^{(-)}\right) / \rho$ in which $-1 \leq \delta \leq 1$, and $\rho=\rho^{(+)}+\rho^{(-)}$is the total density of system.

To study the macroscopic properties of the system, we calculate the total free energy per nucleon, $\mathcal{F}$,

$$
\mathcal{F}=E-T\left(S_{n}^{(+)}+S_{p}^{(+)}+S_{n}^{(-)}+S_{p}^{(-)}\right),
$$

where $E$ is the total energy per nucleon and $S_{j}^{(i)}$ shows the entropy per nucleon for the isospin and spin projection $j$ and $i$, respectively,

$$
\begin{aligned}
S_{j}^{(i)}(\rho, T, B)= & -\frac{1}{A} \sum_{k}\left\{\left[1-n_{j}^{(i)}\left(k, T, \rho_{j}^{(i)}, B\right)\right] \times\right. \\
& \ln \left[1-n_{j}^{(i)}\left(k, T, \rho_{j}^{(i)}, B\right)\right]+ \\
& \left.n_{j}^{(i)}\left(k, T, \rho_{j}^{(i)}, B\right) \ln n_{j}^{(i)}\left(k, T, \rho_{j}^{(i)}, B\right)\right\} .
\end{aligned}
$$

In the above equation, $n_{j}^{(i)}\left(k, T, \rho_{j}^{(i)}, B\right)$ denotes the Fermi-Dirac distribution function,

$$
n_{j}^{(i)}\left(k, T, \rho_{j}^{(i)}, B\right)=\frac{1}{\exp \left(\left[\epsilon_{j}^{(i)}-\mu_{j}^{(i)}\right] / k_{B} T\right)+1},
$$

where $\epsilon_{j}^{(i)}\left(k, T, \rho_{j}^{(i)}, B\right)$ and $\mu_{j}^{(i)}\left(T, \rho_{j}^{(i)}, B\right)$ are the single particle energy and chemical potential, respectively. For a system at temperature $T$ and number density $\rho_{j}^{(i)}$, the chemical potential is specified by

$$
\sum_{k} n_{j}^{(i)}\left(k, T, \rho_{j}^{(i)}, B\right)=N_{j}^{(i)}
$$

Here, we approximate the single particle energy of nucleons with the relations in terms of the effective mass and the momentum independent single particle potential, $U_{j}^{(i)}(\rho, T, B)$, [52, 53]. 
The single particle energy of neutrons is

$$
\begin{aligned}
\epsilon_{n}^{(i)}\left(k, T, \rho_{n}^{(i)}, B\right)= & \frac{\hbar^{2} k^{2}}{2 m_{n}^{*(i)}(\rho, T, B)}-\lambda_{i} \mu_{n} B+ \\
& U_{n}^{(i)}(\rho, T, B)
\end{aligned}
$$

where $\lambda_{ \pm}= \pm 1$ and $\mu_{n}$ is the neutron magnetic moment. For the protons, we also have

$$
\begin{aligned}
\epsilon_{p}^{(i)}\left(k, T, \rho_{p}^{(i)}, B\right)= & \frac{\hbar^{2} k^{2}}{2 m_{p}^{*(i)}(\rho, T, B)}+ \\
& \frac{e \hbar B}{c m_{p}^{*(i)}(\rho, T, B)}\left(l^{(i)}+\frac{1}{2}\right)-\lambda_{i} \mu_{p} B+ \\
& U_{p}^{(i)}(\rho, T, B) .
\end{aligned}
$$

Here, $l^{(i)}=0,1,2,3, \ldots$ are the integers labeling the Landau levels [54] for a proton with spin projection $i, e$ is the proton charge, $c$ is the speed of light, and $\mu_{p}$ is the proton magnetic moment.

In our calculations, we numerically minimize the free energy with respect to the variations in the effective masses, and we obtain the chemical potentials and the effective masses of the spin-up and spin-down nucleons at the minimum point of the free energy.

The total energy of magnetized nuclear matter is calculated using the LOCV method. In this approach, we consider a trial many body wave function of the form

$$
\psi=F \phi
$$

in which $\phi$ is the uncorrelated ground-state wave function of $A$ independent nucleons and $F$ is a proper $A$-body correlation function. Applying Jastrow ansatz [55], $F$ is replaced by

$$
F=S \prod_{i>j} f(i j)
$$

with the symmetrizing operator $S$. The cluster expansion of the energy functional up to the two-body term is as follows,

$$
E([f])=\frac{1}{A} \frac{\langle\psi|H| \psi\rangle}{\langle\psi \mid \psi\rangle}=E_{1}^{p}+E_{1}^{n}+E_{2} .
$$

$E_{1}^{p}$ and $E_{1}^{n}$ are the one-body energies of protons and neutrons, respectively, and $E_{2}$ is the two-body energy. 
At the temperature $T$, the one-body term for the protons, $E_{1}^{p}$, is given by

$$
\begin{aligned}
E_{1}^{p}= & \frac{e B}{\pi h c \rho} \sum_{i=+,-} \int_{0}^{\infty} d k \sum_{l^{(i)}=0}^{\infty} n_{p}^{(i)}\left(k, T, \rho_{p}^{(i)}, B\right) \\
& \times\left(\frac{\hbar^{2} k^{2}}{2 m_{p}}+\frac{e \hbar B}{c m_{p}}\left(l^{(i)}+\frac{1}{2}\right)-\lambda_{i} \mu_{p} B\right),
\end{aligned}
$$

where $n_{p}^{(i)}$ has been given in Eq. (3) . Moreover, the one-body term for the neutrons, $E_{1}^{n}$, is as follows,

$$
E_{1}^{n}=\sum_{i=+,-} \sum_{k} n_{n}^{(i)}\left(k, T, \rho_{n}^{(i)}, B\right)\left(\frac{\hbar^{2} k^{2}}{2 m_{n}}-\lambda_{i} \mu_{n} B\right),
$$

with $n_{n}^{(i)}$ given in Eq. (3).

The two-body energy, $E_{2}$, is

$$
E_{2}=\frac{1}{2 A} \sum_{i j}\langle i j|\nu(12)| i j-j i\rangle,
$$

where

$$
\begin{aligned}
\nu(12)= & -\frac{\hbar^{2}}{2 m}\left[f(12),\left[\nabla_{12}^{2}, f(12)\right]\right]+ \\
& f(12) V(12) f(12) .
\end{aligned}
$$

Here, $V(12)$ denotes the nuclear potential which in this study, the $A V_{18}$ potential is substituted [56]. In addition, $f(12)$ presents the two-body correlation function with the form $f(12)=\sum_{k=1}^{3} f^{(k)}\left(r_{12}\right) P_{12}^{(k)}$ with $P_{12}^{(k)}$ given in Ref. [48]. The two-body energy can be obtained using the two-body correlation function and the nuclear potential. Afterwards, the two-body energy is minimized with respect to the variations in the functions $f^{(i)}$ subject to the normalization constraint, $\frac{1}{A} \sum_{i j}\left\langle i j\left|h_{S_{z}}^{2}-f^{2}(12)\right| i j\right\rangle_{a}=0$, with the Pauli function $h_{S_{z}}(r)$ as follows [48],

$$
h_{S_{z}}(r)= \begin{cases}{\left[1-\frac{1}{2}\left(\frac{\gamma^{(i)}(r)}{\rho}\right)^{2}\right]^{-1 / 2}} & ; S_{z}= \pm 1 \\ 1 & ; S_{z}=0\end{cases}
$$

where

$$
\gamma^{(i)}(r)=\frac{1}{\pi^{2}} \int n^{(i)}\left(k, T, \rho^{(i)}, B\right) J_{0}(k r) k^{2} d k .
$$

Solving the differential equations resulted from the minimization procedure leads to the correlation functions and the two-body energy term. In the next step, it is possible to calculate the free energy of nuclear matter using Eqs. (11) and (9). 


\section{RESULTS AND DISCUSSION}

To investigate the properties of hot magnetized nuclear matter, we have presented the free energy of system as a function of the density at different temperatures and magnetic fields in Figs. 1 and 2. It is obvious that for all given values of the magnetic field and temperature the nuclear matter is self-bound. However, the density at which the nuclear matter saturates and the corresponding free energy depend on the temperature and magnetic field (as we will discuss these dependencies in more details). As we can see from Figs. 1 and 2, the influences of the temperature and magnetic field on the free energy are more considerable at lower densities. This is due to the larger contribution of nuclear energy at higher densities. In addition, Fig. 2 indicates that the magnetic field can affect the nuclear matter more significantly at lower temperatures. Fig. 3 shows the density and temperature dependence of the nuclear matter spin polarization parameter for different magnetic fields. It is clear that the spin polarization parameter of the system decreases as density grows. In addition, the spin polarization parameter of the nuclear matter has smaller values at higher temperatures, while it increases by increasing the magnetic field. The effects of temperature and magnetic field are more significant at lower densities.

\section{A. Symmetry energy}

For nuclear matter, the symmetry energy which quantifies the produced energy when neutron matter converts to symmetric nuclear matter is

$$
E_{\text {sym }}=\left.\frac{1}{2} \frac{\partial^{2} E}{\partial \eta^{2}}\right|_{\eta=0},
$$

where $\eta=(N-Z) /(N+Z)$ is the isospin asymmetry parameter. In the parabolic approximation, the symmetry energy is given by [58]

$$
E_{\text {sym }}=E_{\text {neut }}-E_{\text {nucl }} \text {. }
$$

In the above equation, $E_{\text {neut }}$ and $E_{\text {nucl }}$ denote the energy per particle of the pure neutron matter and symmetric nuclear matter. Figs. 4 and 5 present the symmetry energy of hot magnetized nuclear matter at different temperatures and magnetic fields. It can be seen that the symmetry energy grows as density increases. In addition, it is obvious from Fig. 4 that at each magnetic field and density, the symmetry energy decreases as the temperature 
increases. This result is in agreement with the results of Ref. [14]. Fig. 5 confirms that the symmetry energy decreases with the increase in magnetic field. This quantity is more affected by the magnetic field at lower densities. Figs. 4 and 5 show that the slope of the symmetry energy is larger at higher magnetic fields, while the temperature has a negligible effect on the slope of symmetry energy. Moreover, the results show that at high magnetic fields and low densities, the symmetry energy is negative. This is due to the interaction of nucleons magnetic moment and magnetic field.

\section{B. Saturation properties}

We have plotted the saturation density of magnetized nuclear matter as a function of temperature for different magnetic fields in Fig. 6. It is seen that at each magnetic field, the saturation density decreases by increasing the temperature. It means that the nuclear matter at higher temperatures saturates at lower values of the density. However, we can see that at low temperatures for $B>10^{18} G$, the saturation density increases as the magnetic field grows. This result, for the nuclear matter at zero temperature, has been also reported in our previous study of cold magnetized nuclear matter [50]. This increase is due to the fact that in the cold magnetized nuclear matter, the contribution of magnetic energy is significant and it prevents the nuclear matter from the saturation. Therefore, in such conditions, the higher values of density are needed to saturate the nuclear matter. This is while for $T>15 \mathrm{MeV}$, the saturation density decreases by increasing the magnetic field. This result indicates that in the nuclear matter at enough high temperatures, the interaction of nucleons with the magnetic field helps the system to saturate at smaller densities.

Fig. 7 presents the value of free energy at the saturation point versus the temperature at different magnetic fields. It is obvious that this value of free energy decreases by increasing the temperature. At each temperature, for $B>10^{18} G$, the free energy at the saturation point increases with the increase in magnetic field. This result also holds for cold nuclear

matter [50]. This can be interpreted by the significant contribution of the magnetic energy at stronger magnetic fields. Another result of this work is the behavior of the nuclear matter spin polarization parameter at the saturation point which has presented in Fig. 8. At each magnetic field, by increasing the temperature, the spin polarization parameter of system grows. This is a consequence of the smaller values of saturation density at higher 
temperatures (Fig. 6) and the larger values of the nuclear matter spin polarization parameter at lower temperatures (Fig. 3). In addition, the spin polarization parameter of nuclear matter increases by increasing the magnetic field.

The isothermal incompressibility at the saturation point is given by

$$
\mathcal{K}(B, T)=9 \rho_{0}^{2}(B, T)\left[\frac{\partial^{2} \mathcal{F}(\rho, B, T)}{\partial \rho^{2}}\right]_{\rho=\rho_{0}(B, T)} .
$$

Fig. 9 shows our results for the isothermal incompressibility of magnetized nuclear matter. At each magnetic field, the isothermal incompressibility reduces by increasing the temperature. Previously, we have reported this result for zero magnetic field in Ref. [57]. It can be found from this result that as the temperature increases, the number of accessible states for the nucleons grows leading to more compressible nuclear matter. However, it is clear from Fig. 9 that at low temperatures and for $B>10^{18} G$, the isothermal incompressibility increases by increasing the magnetic field. This indicates the stiffening of equation of state (EOS) at high magnetic fields. In fact, at low temperatures for $B>10^{18} G$, the softening of EOS is overwhelmed by stiffening due to the spin polarization parameter of nuclear matter, as in our previous study of cold magnetized nuclear matter [50]. However, at high temperatures, the isothermal incompressibility decreases as the magnetic field increases. This confirms the softening of EOS at high magnetic fields. We can conclude that at high temperatures and magnetic fields, the Landau quantization is the dominant effect which leads to the softening of EOS.

\section{Equation of state}

The equation of state for magnetized nuclear matter at different temperatures and magnetic fields can be calculated using the following relation,

$$
P(\rho, T, B)=\rho^{2}\left(\frac{\partial F(\rho, T, B)}{\partial \rho}\right)_{T, B} .
$$

Our results at four temperatures and three magnetic fields have presented in Fig. 10, It is obvious that at zero temperature, the pressure is negative below the saturation density. Besides, this quantity is zero at the saturation point. For all magnetic fields, the range

of density at which the pressure is negative reduces as the temperature grows. This holds until the pressure becomes a positive definite function at a specific point. The temperature 
corresponding to this point is called flashing temperature, $T_{f}$ [28]. In fact, above the flashing temperature, the kinetic energy is more significant than the binding energy of the nuclear matter, and the increase of temperature makes the nuclear matter unbound. Besides, the flashing density, $\rho_{f}$, which satisfies the constraints,

$$
P\left(\rho=\rho_{f}\right)=\left(\frac{\partial P}{\partial \rho}\right)_{\rho_{f}}=0
$$

characterizes the flashing point. The physical importance of the flashing point is due to the fact that it corresponds to the maximum temperature of the nuclear matter at which the system is self-bound [28]. The nuclear matter at the flashing point is at zero pressure. Because of the positive pressure of nuclear matter at higher temperatures, i.e. $T>T_{f}$, the increasing of temperature from the flashing temperature leads to the expansion of nuclear matter. The properties of the flashing point at different magnetic fields are presented in Table I. We can see that the flashing temperature, with a value between 17.0 and $17.5 \mathrm{MeV}$ for the given magnetic fields, decreases as the magnetic field grows. It is due to the fact that besides the temperature, the increase of the magnetic field makes the system less bound. Therefore, for magnetized nuclear matter at high magnetic fields, the low temperatures are needed to make the system unbound. Our result for the flashing density, i.e. $0.19 \mathrm{fm}^{-3}$, indicates that this quantity is not sensitive to the magnitude of magnetic field (see Table II). Moreover, our flashing density is greater than the half of our saturation density at zero temperature and magnetic field, i.e. $0.310 \mathrm{fm}^{-3}$ [58] which is similar to that of others [23, 28].

By increasing the temperature above the flashing point, the isotherms are positive at each density. However, at some densities, these isotherms are decreasing function of the density, i.e. $\partial P / \partial \rho<0$. This holds until we reach the critical temperature, $T_{c}$. For the critical temperature and the temperatures above that, the pressure grows monotonically as the density increases. We have presented the critical isotherm in Fig. 10, The value of the critical density, $\rho_{c}$, can be found by the following constraints,

$$
\left(\frac{\partial P}{\partial \rho}\right)_{\rho_{c}}=\left(\frac{\partial^{2} P}{\partial \rho^{2}}\right)_{\rho_{c}}=0 .
$$

The critical properties of magnetized nuclear matter will be discussed in the following. Fig. 10 also shows an isotherm above the critical point, i.e. $\mathrm{T}=30 \mathrm{MeV}$. 


\section{Liquid gas phase transition}

Fig. 11 gives the equation of state at different temperatures and magnetic fields. We can find from this figure that at low temperatures and densities, the nuclear matter is mechanically unstable. This leads to a first order liquid gas phase transition in the nuclear matter. It is clear that the isotherms present a typical Van der Waals like behavior in which the liquid and gaseous phases coexist. It is possible to find the densities of gas and liquid at each temperature applying the equal-area Maxwell construction. Fig. 12 shows the coexistence curves, i.e. the liquid and gas densities versus temperature at different magnetic fields. It is clear that the liquid density decreases and the gas density increases by increasing the temperature. Inside the coexistence curve, the stable phase of nuclear matter is a mixture of liquid and gas. The coexistence region reduces as the temperature increases, and it disappears at the critical temperature. At this point, the densities of gas and liquid become equal and the nuclear matter experiences a second-order phase transition. It is clear that the coexistence region reduces as the magnetic field grows. In addition, at high magnetic fields, the coexistence region disappears at lower temperatures. This shows that the critical temperature reduces by increasing the magnetic field. Fig. 12 also indicates that the effect of magnetic field on the coexistence curve is more significant at higher temperatures. The critical properties of magnetized nuclear matter at different magnetic fields have been given in Table II] We can see that by increasing the magnetic field from $10^{18}$ to $10^{19} \mathrm{G}$, the critical temperature decreases from 22.9 to $21.8 \mathrm{MeV}$. In addition, the critical pressure decreases from $0.93 \mathrm{MeV} \mathrm{fm}^{-3}$ to $0.84 \mathrm{MeV} \mathrm{fm}^{-3}$ by increasing the magnetic field from $10^{18} \mathrm{G}$ to $10^{19} G$. However, the critical densities are nearly identical for different magnetic fields. According to the results given in Table II, it can be easily seen that the value of the ratio $\gamma_{c}=P_{c} /\left(\rho_{c} T_{c}\right)$ is $0.29,0.28$, and 0.28 for the magnetic fields $10^{18}, 5 \times 10^{18}$, and $10^{19} G$, respectively, which are close to the value $\gamma_{c} \approx 0.28$ [23, 28]. Besides, Table II] shows that as the magnetic field increases, the critical temperature and pressure decrease toward the experimental values $T_{c}=17.9 \mathrm{MeV}$ and $P_{c}=0.31 \mathrm{MeV} \mathrm{fm}^{-3}$.

The difference in the liquid and gas densities denotes the order parameter of the liquid gas phase transition. We define the order parameter as $m=\rho_{\text {liquid }}-\rho_{\text {gas }}$ to show the behavior of liquid gas phase transition in the nuclear matter. Fig. 13 presents the order parameter versus the temperature at different magnetic fields. It can be seen that for each magnetic 
field, the order parameter vanishes at the critical temperature. Below the critical point, the order parameter decreases as the magnetic field grows. The effects of the magnetic field on the order parameter is more important at higher temperatures.

\section{SUMMARY AND CONCLUDING REMARKS}

The microscopic nuclear potential, $A V_{18}$, and LOCV method were applied to investigate the properties of symmetric nuclear matter in the presence of strong magnetic fields at finite temperature, the conditions which are found in protoneutron stars and heavy ion collisions. The free energy of nuclear matter versus the density at different temperatures and magnetic fields shows that the nuclear matter is self-bound at low enough temperatures. We have shown that the effect of the temperature and magnetic field on the free energy is more considerable at lower densities. Moreover, due to the contribution of strong interaction at high densities, the effect of the magnetic field on the nuclear matter is more significant at lower

temperatures. It has been shown that the symmetry energy decreases by increasing the temperature and magnetic field. Our results indicate that the saturation density decreases by increasing the temperature. However, the effect of the magnetic field on the saturation density depends on the temperature. At lower temperatures, the magnetic field increases the saturation density, while it decreases the saturation density for the system at higher temperatures. In addition, we found that the free energy corresponding to the saturation point decreases as the temperature grows. Besides, the free energy at the saturation point increases with the increase in magnetic field. The spin polarization parameter of nuclear matter at the saturation density is an increasing function of the temperature. This enchantment is the result of the lower values of the saturation density at higher temperatures. It was clarified that the isothermal incompressibility is a decreasing function of the temperature. The increase in the magnetic field leads to the stiffening of EOS at lower temperatures, while at higher temperatures, any increase in the magnetic field results in the softening of EOS. We have also studied the equation of state for magnetized nuclear matter. It has been shown that the flashing temperature which corresponds to the maximum temperature of the nuclear matter at which the system is self-bound, decreases as the magnetic field grows. However, our results confirm that the flashing density is not sensitive to the magnetic field. The equation of state for magnetized nuclear matter also shows that a first order liquid 
gas phase transition takes place in the nuclear matter. We have calculated the coexistence curves for the liquid gas phase transition at different magnetic fields. We have found that the coexistence region reduces as the magnetic field grows. The critical properties of magnetized nuclear matter at different magnetic fields have also been calculated. The results indicate that the critical temperature and pressure decrease by increasing the magnetic field. Finally, we have presented the order parameter of the liquid gas phase transition. It was shown that this quantity decreases as the magnetic field increases. Our results for the symmetric nuclear matter have important consequences on hot dense magnetized nuclear systems in protoneutron stars and relativistic heavy ion collisions.

\section{Acknowledgments}

This work has been supported financially by the Center for Excellence in Astronomy

and Astrophysics (CEAA-RIAAM). We also wish to thank the Shiraz University Research Council.

[1] G. F. Chapline, M. H. Johnson, E. Teller, M. S. Weiss, Phys. Rev. D 8, 4302 (1973).

[2] M. Camenzind, Compact Objects in Astrophysics: White Dwarfs, Neutron Stars and Black Holes (Springer-Verlag, Berlin, Heidelberg, 2007).

[3] Y. F. Yuan, J. L. Zhang, Astron. Astrophys. 335, 969 (1998).

[4] M. Barranco, J.-R. Buchler, Phys. Rev. C 24, 1191 (1981).

[5] C. Das, R. K. Tripathi, R. Sahu, Phys. Rev. C 45, 2217 (1992).

[6] H. Kanzawa, K. Oyamatsu, K. Sumiyoshi, M. Takano, Nucl. Phys. A 791, 232 (2007).

[7] N. M. Ghulam, H. B. Ghassib, M. K. Al-Sugheir, Phys. Rev. C 75, 064317 (2007).

[8] A. Mukherjee, Phys. Rev. C 79, 045811 (2009).

[9] O. N. Ghodsi, R. Gharaei, Phys. Rev. C 84, 024612 (2011).

[10] H. Pais, A. Sulaksono, B. K. Agrawal, C. Providncia, Phys. Rev. C 93, 045802 (2016).

[11] C. Mondal, B. K. Agrawal, J. N. De, S. K. Samaddar, Phys. Rev. C 93, 044328 (2016).

[12] N. Alam, B. K. Agrawal, M. Fortin, H. Pais, C. Providncia, Ad. R. Raduta, A. Sulaksono, Phys. Rev. C 94, 052801 (2016). 
[13] Ngo Hai Tan, Doan Thi Loan, Dao T. Khoa, Jerome Margueron, Phys. Rev. C 93, 035806 (2016).

[14] A.N. Antonov, D.N. Kadrev, M.K. Gaidarov, P. Sarriguren, E. Moya de Guerra, accepted for publication in Physical Review C, arXiv:1702.00576.

[15] J. B. Natowitz et al., Phys. Rev. Lett. 89, 212701 (2002).

[16] J. B. Elliott, P. T. Lake, L. G. Moretto, L. Phair, Phys. Rev. C 87, 054622 (2013).

[17] B. Friedman, V. R. Pandharipande, Nucl. Phys. A 361, 502 (1981).

[18] M. Malheiro, A. Delfino, C. T. Coelho, Phys. Rev. C 58, 426 (1998).

[19] M. Baldo, L. S. Ferreira, Phys. Rev. C 59, 682 (1999).

[20] M. Abd-Alla, S. A. Hager, Phys. Rev. C 61, 044313 (2000).

[21] W. Zuo, Z. H. Li, A. Li, G. C. Lu, Phys. Rev. C 69, 064001 (2003).

[22] W. Zuo, Z. H. Li, A. Li, U. Lombardo, Nucl. Phys. A 745, 34 (2004).

[23] A. Rios, Nucl. Phys. A 845, 58 (2010).

[24] B. K. Sharma, S. Pal, Phys. Rev. C 81, 064304 (2010).

[25] G. H. Zhang, W. Z. Jiang, Phys. Lett. B 720, 148 (2013).

[26] A. Rios, A. Polls, A. Ramos, H. Mther, Phys. Rev. C 78, 044314 (2008).

[27] C. Wellenhofer, J. W. Holt, N. Kaiser, W. Weise, Phys. Rev. C 89, 064009 (2014).

[28] A. Rios, X. R. Maza, J. Phys. G: Nucl. Part. Phys. 42, 034005 (2015).

[29] S. Chakrabarty, D. Bandyopadhyay, S. Pal, Phys. Rev. Lett. 78, 2898 (1997).

[30] A. Rabhi, C. Providencia, J. Da Providencia, Phys. Rev. C 79, 015804 (2009).

[31] J.P.W. Diener, F.G. Scholtz, Phys. Rev. C 87, 065805 (2013).

[32] J. Dong, U. Lombardo, W. Zuo, H. Zhang, Nucl. Phys. A 898, 32 (2013).

[33] A. Haber, F. Preis, A. Schmitt, Phys. Rev. D 90, 125036 (2014).

[34] R. Aguirre, E. Bauer, J. Phys. G: Nucl. Part. Phys. 42, 105101 (2015).

[35] J. C. Owen, R. F. Bishop, J. M. Irvine, Ann. Phys. (NY), 102, 170 (1976).

[36] J. C. Owen, R. F. Bishop, J. M. Irvine, Nucl. Phys. A 274, 108 (1976).

[37] J. C. Owen, R. F. Bishop, J. M. Irvine, Nucl. Phys. A 277, 45 (1977).

[38] M. Modarres, J. Phys. G: Nucl. Phys. 19, 1349 (1993).

[39] M. Modarres, J. Phys. G: Nucl. Phys. 21, 351 (1995).

[40] M. Modarres, J. Phys. G: Nucl. Phys. 23, 923 (1997).

[41] H. R. Moshfegh, M. Modarres, J. Phys. G: Nucl. Phys. 24, 821 (1998). 
[42] G. H. Bordbar, M. Modarres, J. Phys. G: Nucl. Phys. 23, 1631 (1997).

[43] E. Feenberg, Theory of Quantum Fluids, Academic Press, New York (1969).

[44] G. H. Bordbar, M. Bigdeli, Phys. Rev. C 75, 045804 (2007).

[45] G. H. Bordbar, M. Bigdeli, Phys. Rev. C 76, 035803 (2007).

[46] G. H. Bordbar, M. Bigdeli, Phys. Rev. C 77, 015805 (2008).

[47] G. H. Bordbar, M. Bigdeli, Phys. Rev. C 78, 054315 (2008).

[48] M. Bigdeli, G. H. Bordbar, Z. Rezaei, Phys. Rev. C 80, 034310 (2009).

[49] Z. Rezaei, M. Bigdeli, G. H. Bordbar, Int. J. Mod. Phys. E 24, 1550075 (2015).

[50] Z. Rezaei, G. H. Bordbar, Eur. Phys. J. A 52, 132 (2016).

[51] G. H. Bordbar, Z. Rezaei, Rom. J. Phys. 61, 413 (2016).

[52] A. Rios, A. Polls, I. Vidana, Phys. Rev. C 71, 055802 (2005).

[53] D. Lopez-Val, A. Rios, A. Polls, I. Vidana, Phys. Rev. C 74, 068801 (2006).

[54] R. K. Pathria, P. D. Beale, Statistical Mechanics, Pergamon Press (2011).

[55] J. W. Clark, Prog. Part. Nucl. Phys. 2, 89 (1979).

[56] R. B. Wiringa, V. G. J. Stoks, R. Schiavilla, Phys. Rev. C 51, 38 (1995).

[57] M. Modarres, G. H. Bordbar, Phys. Rev. C 58, 2781 (1998).

[58] G. H. Bordbar, M. Modarres, Phys. Rev. C 57, 714 (1998). 
TABLE I: The flashing temperature, $T_{f}$, and density, $\rho_{f}$, for magnetized nuclear matter at different magnetic fields.

\begin{tabular}{|c|c|c|}
\hline$B(G)$ & $T_{f}(\mathrm{MeV})$ & $\rho_{f}\left(\mathrm{fm}^{-3}\right)$ \\
\hline $10^{18}$ & 17.5 & 0.19 \\
$5 \times 10^{18}$ & 17.4 & 0.19 \\
$10^{19}$ & 17.0 & 0.19 \\
\hline
\end{tabular}

TABLE II: The critical temperature, $T_{c}$, density, $\rho_{c}$, and pressure, $p_{c}$, for magnetized nuclear matter at different magnetic fields.

\begin{tabular}{|c|c|c|c|}
\hline$B(G)$ & $T_{c}(\mathrm{MeV})$ & $\rho_{c}\left(\mathrm{fm}^{-3}\right)$ & $p_{c}\left(\mathrm{MeVfm}^{-3}\right)$ \\
\hline $10^{18}$ & 22.9 & 0.14 & 0.93 \\
$5 \times 10^{18}$ & 22.6 & 0.14 & 0.90 \\
$10^{19}$ & 21.8 & 0.14 & 0.84 \\
Experimental result [16] & 17.9 & 0.06 & 0.31 \\
\hline
\end{tabular}



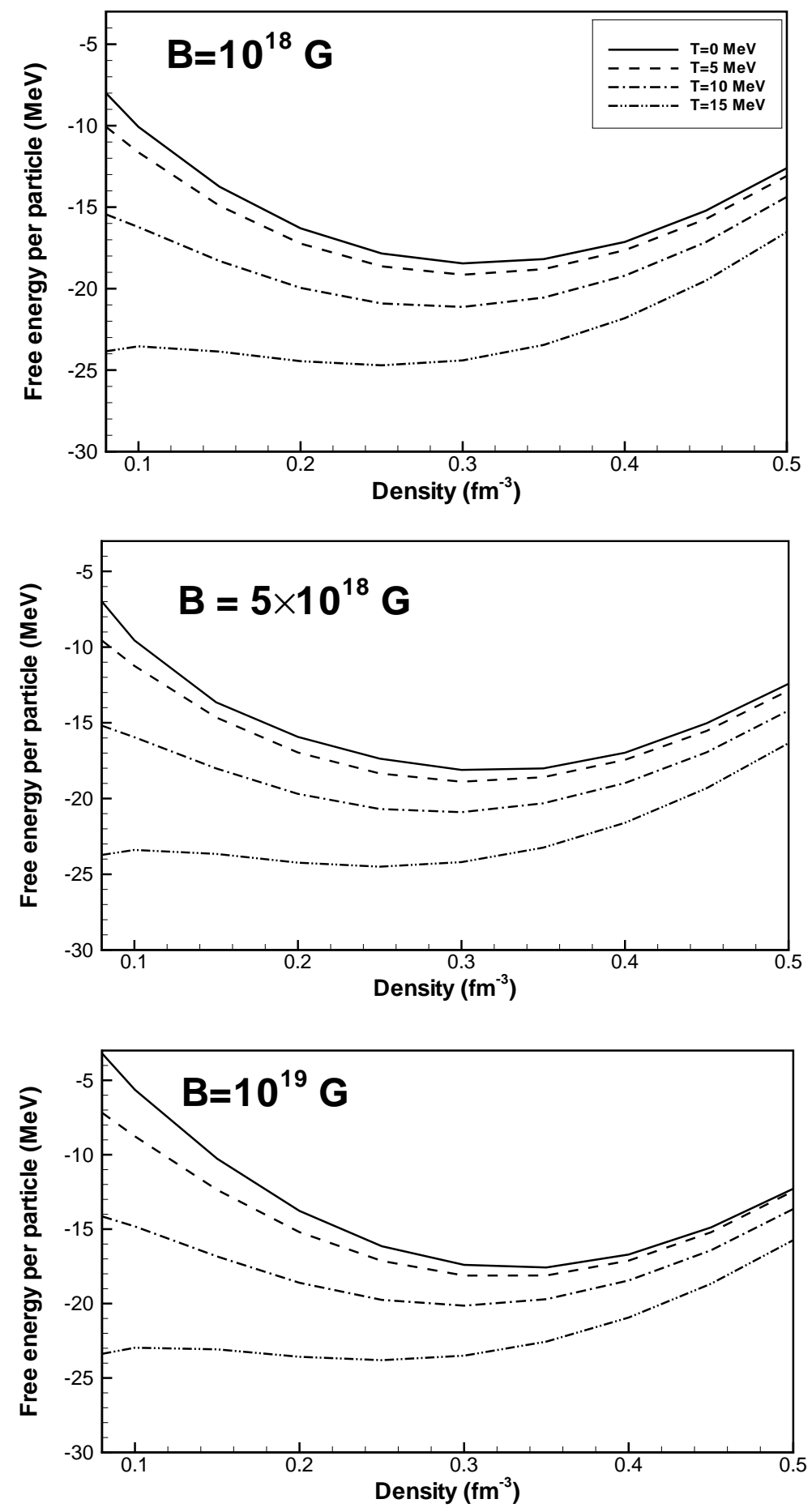

18

FIG. 1: Free energy per particle versus the density at different temperatures and magnetic fields. 

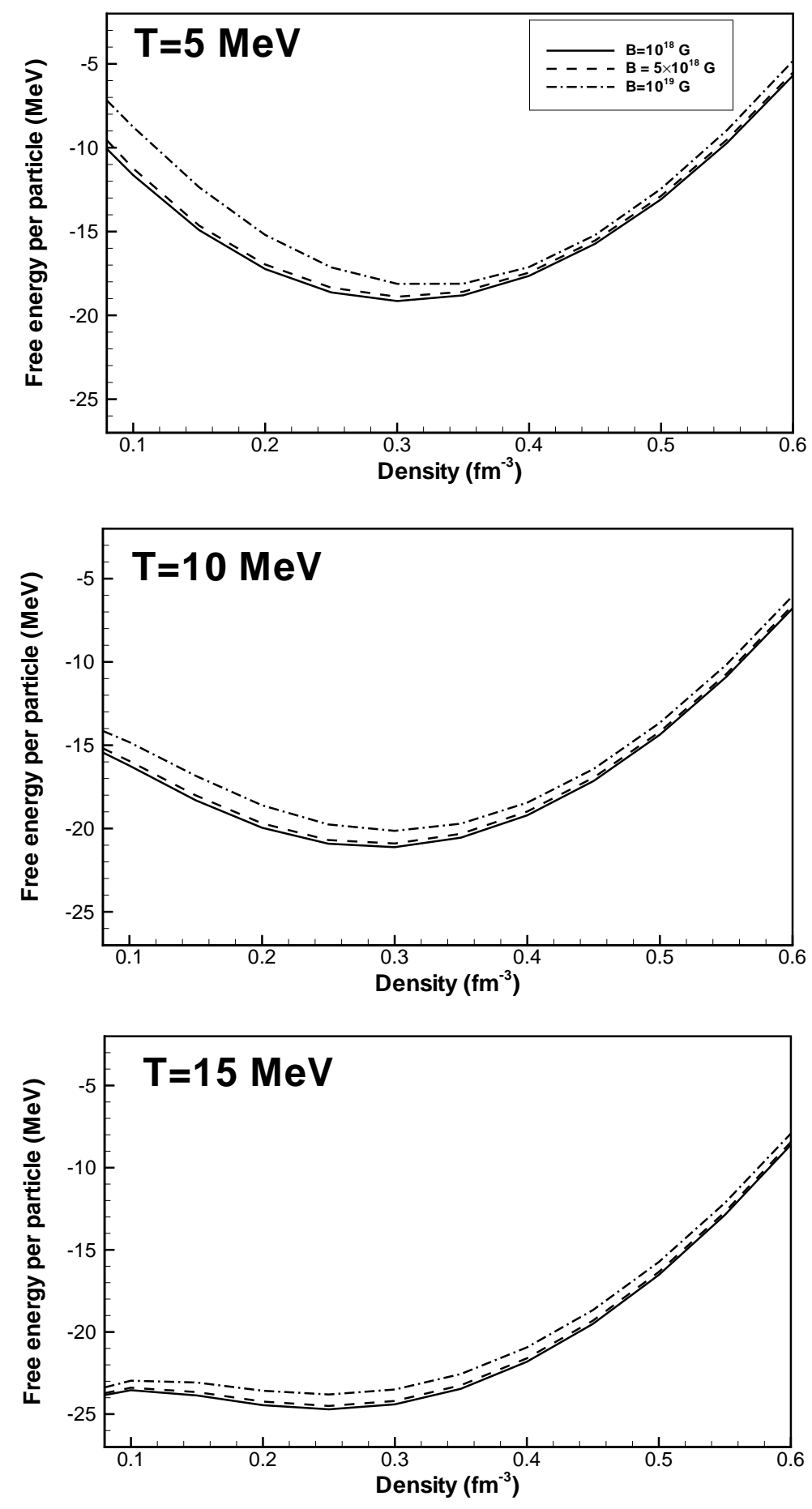

19

FIG. 2: Same as Fig. 1 but for the comparison of free energies at different magnetic fields. 

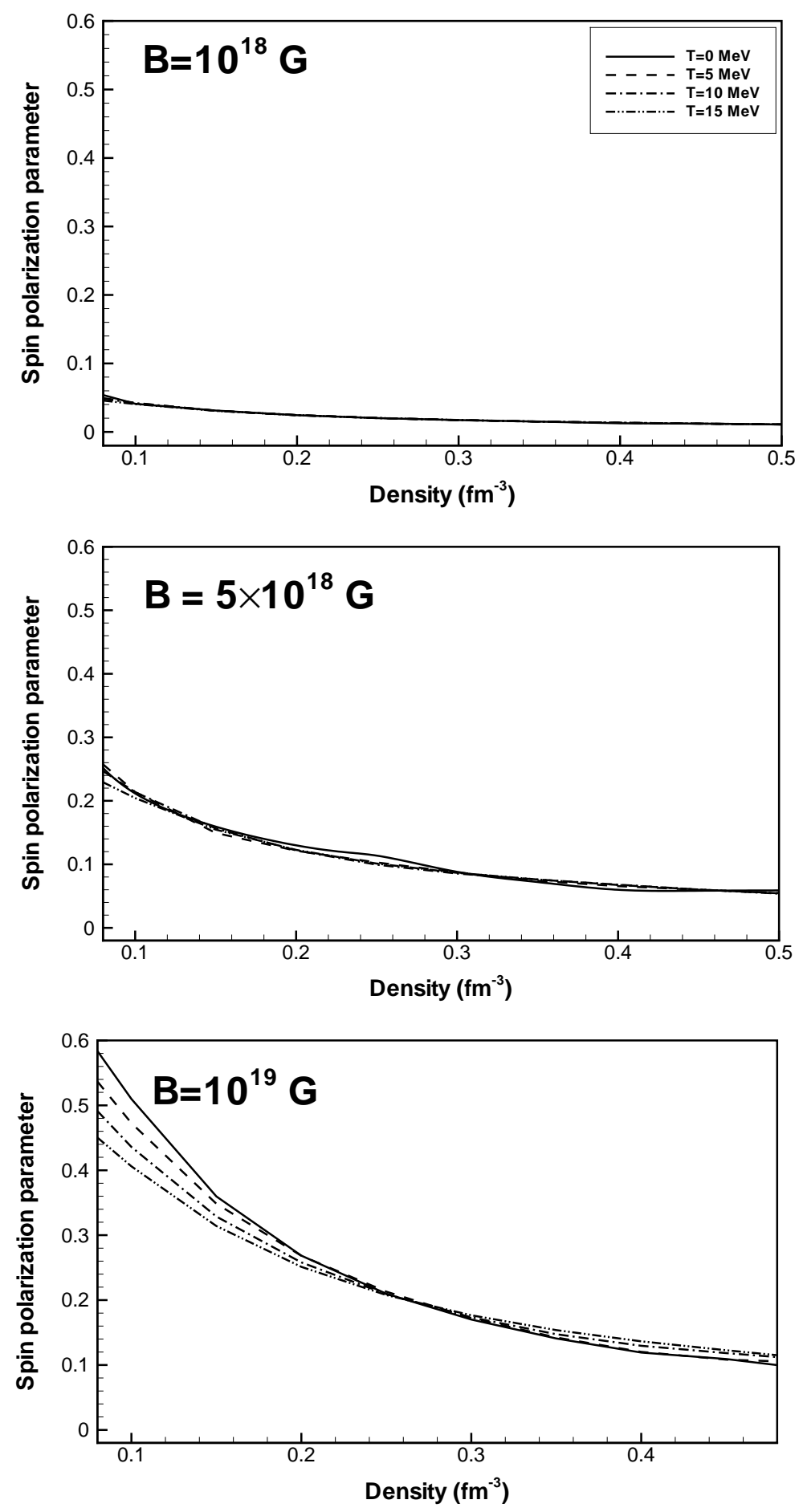

20

FIG. 3: Same as Fig. 1 but for the spin polarization parameter. 

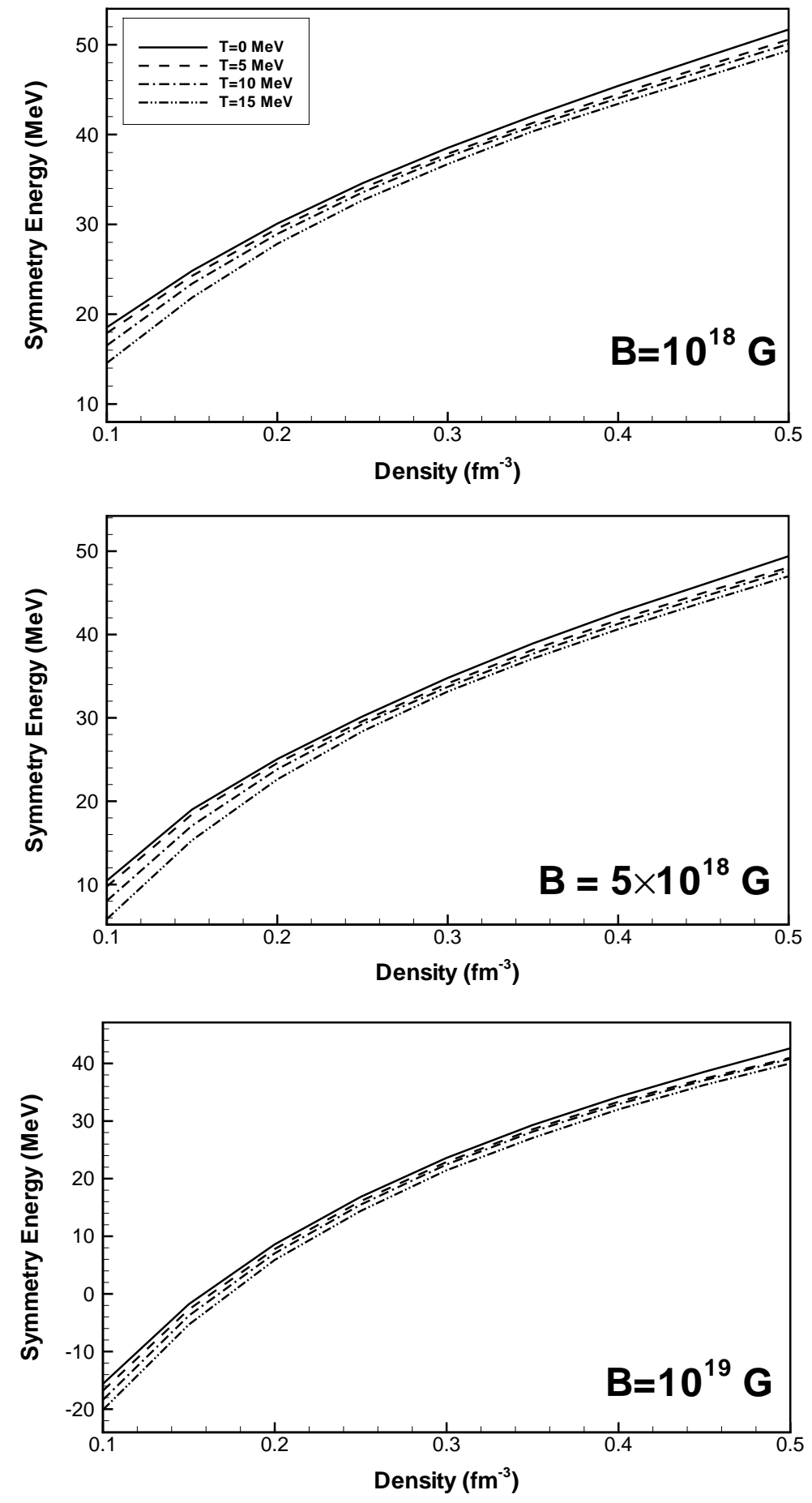

21

FIG. 4: Same as Fig. 1 but for the symmetry energy. 

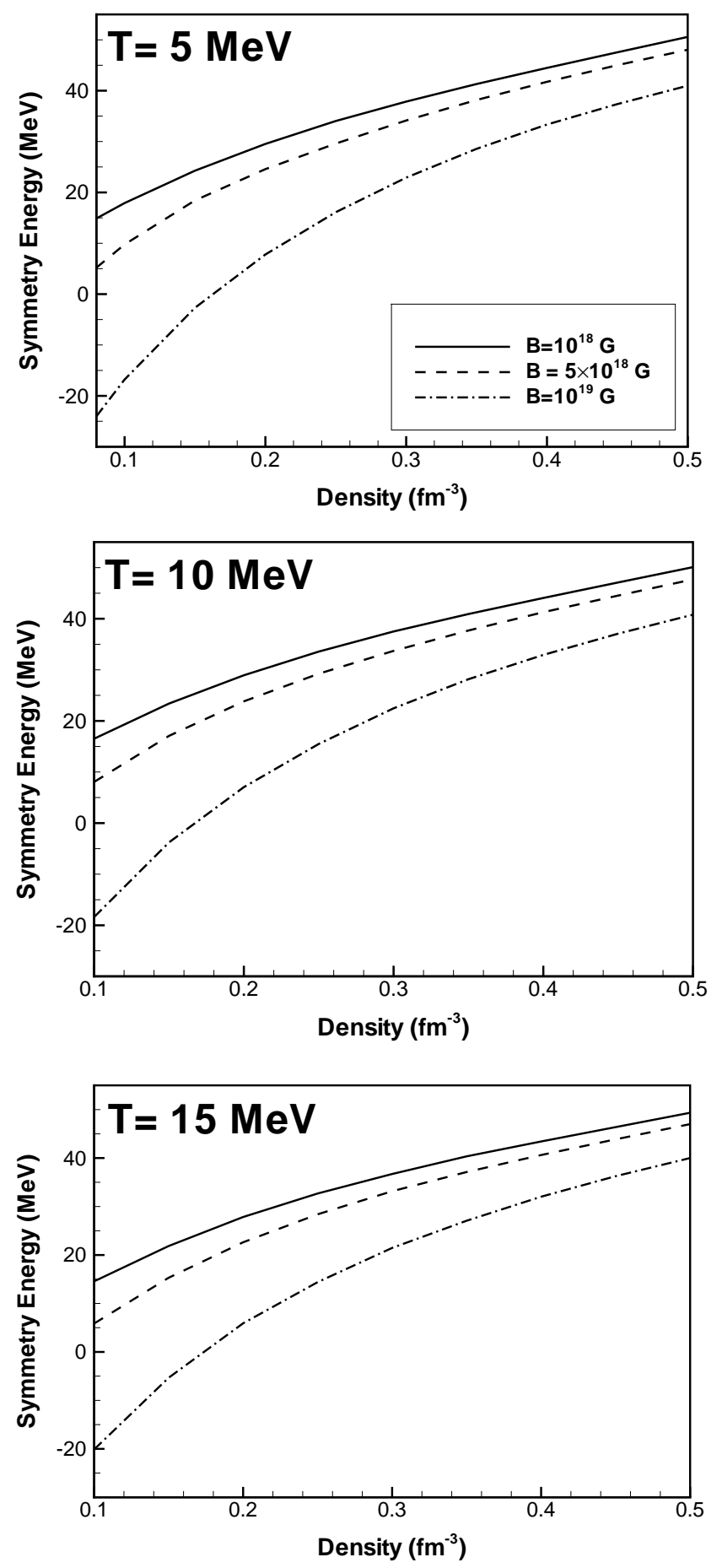

22

FIG. 5: Same as Fig. 2 but for the symmetry energy. 


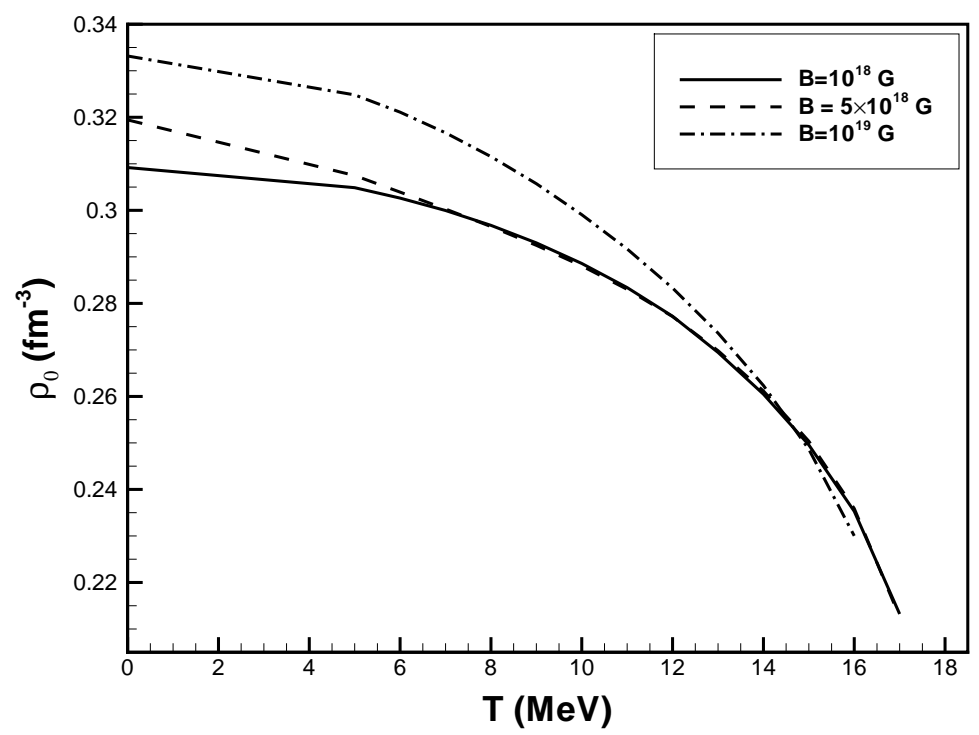

FIG. 6: Saturation density as a function of temperature at different magnetic fields. 


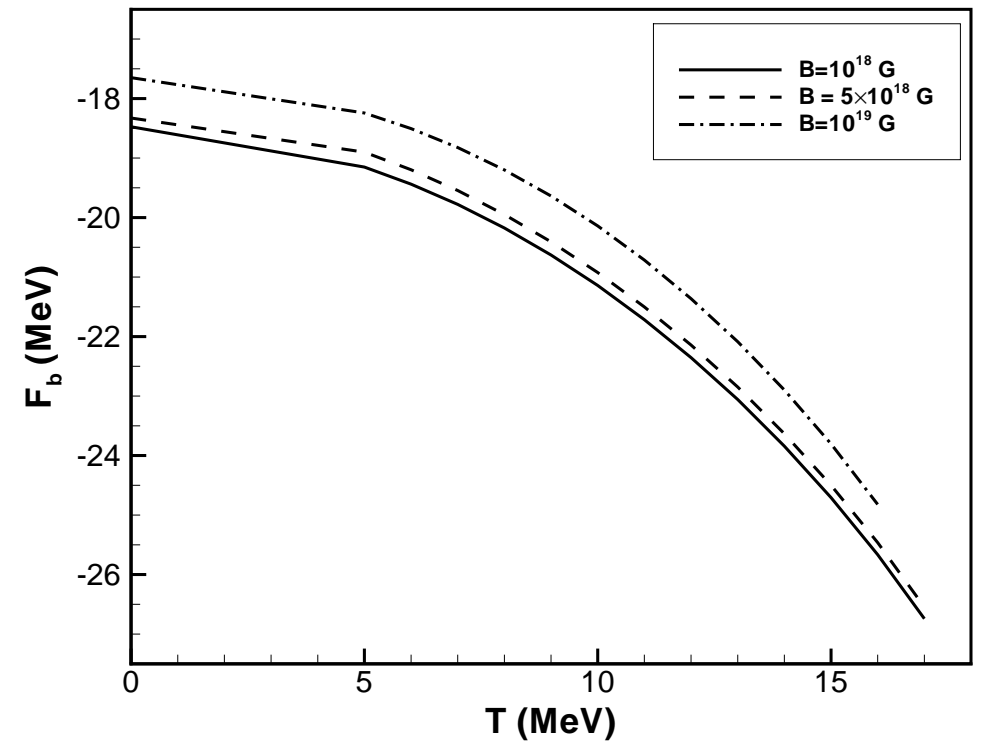

FIG. 7: Temperature dependence of free energy per particle corresponding to the saturation point at different values of magnetic field. 


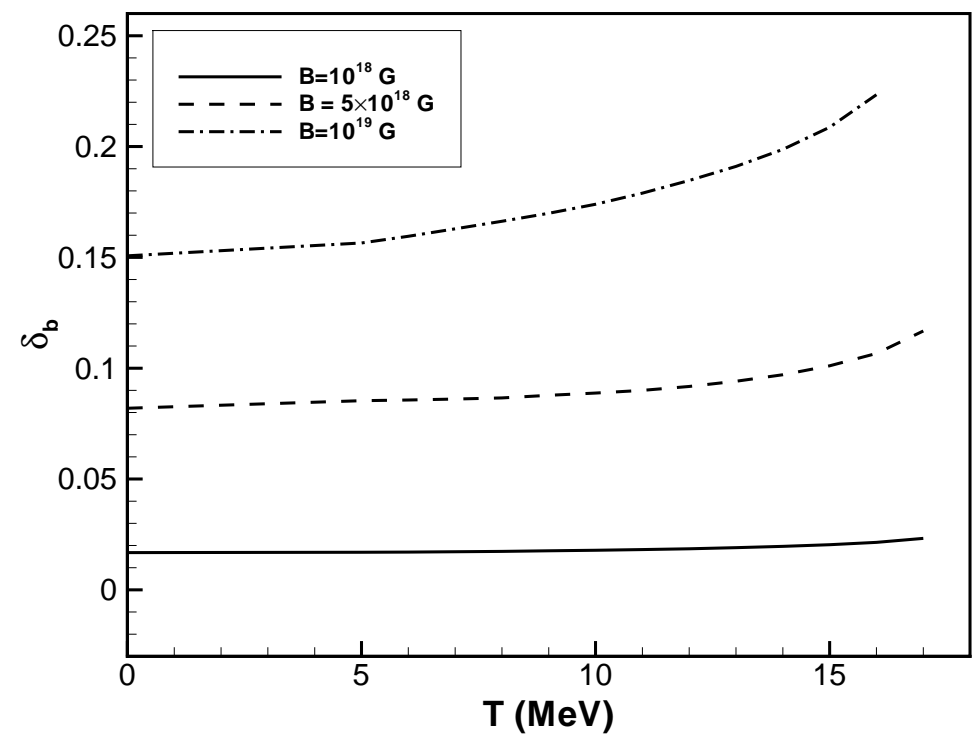

FIG. 8: Same as Fig. 7 but for the spin polarization parameter. 


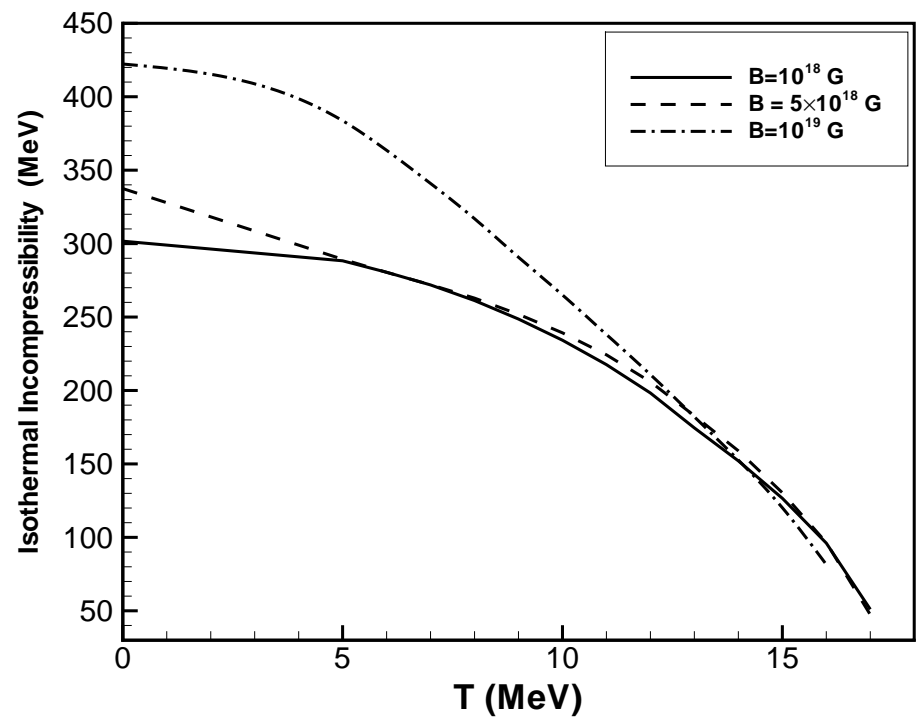

FIG. 9: Same as Fig. 7 but for the isothermal incompressibility. 

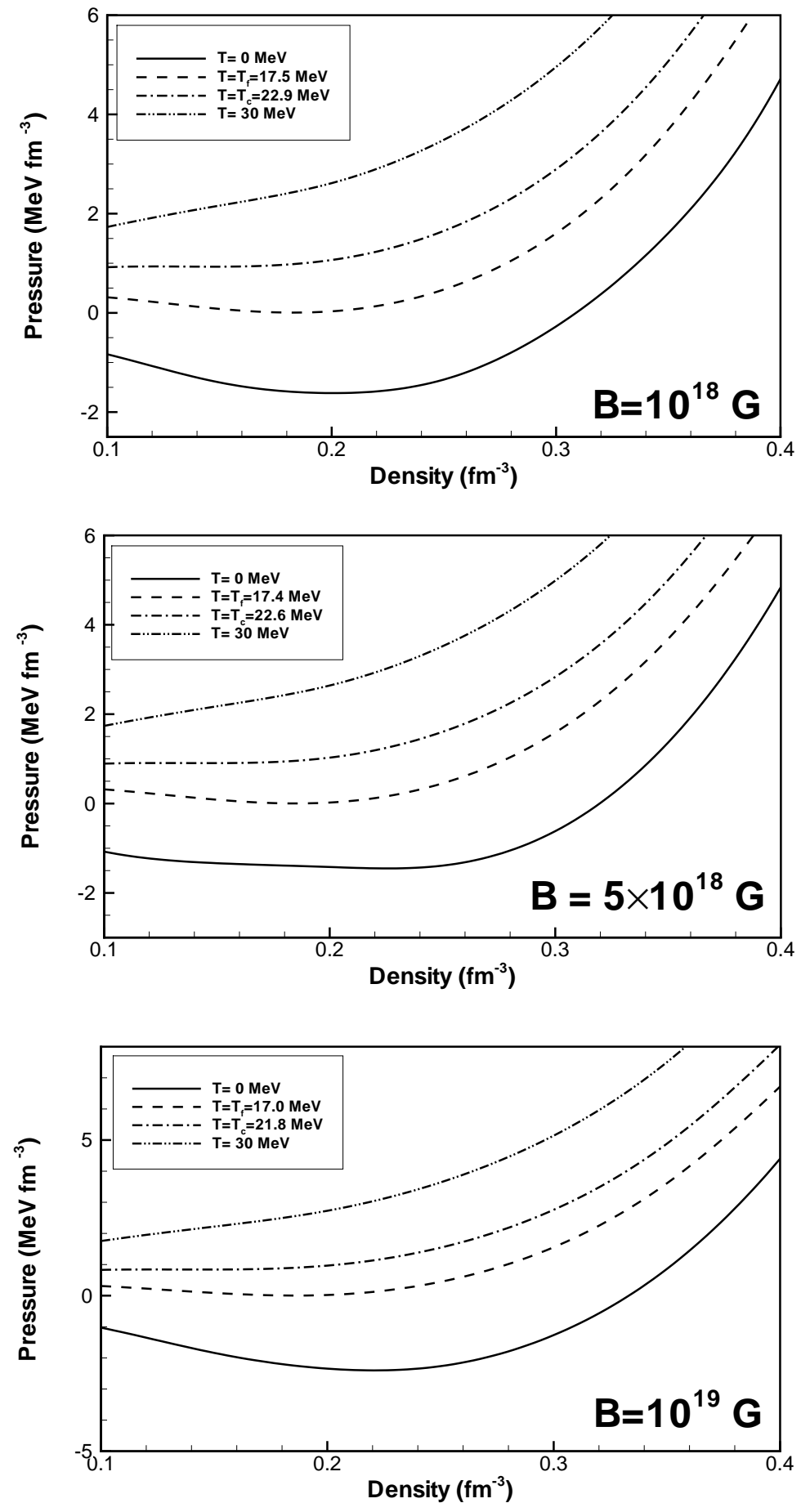

27

FIG. 10: The equation of state of magnetized nuclear matter at different temperatures and magnetic 

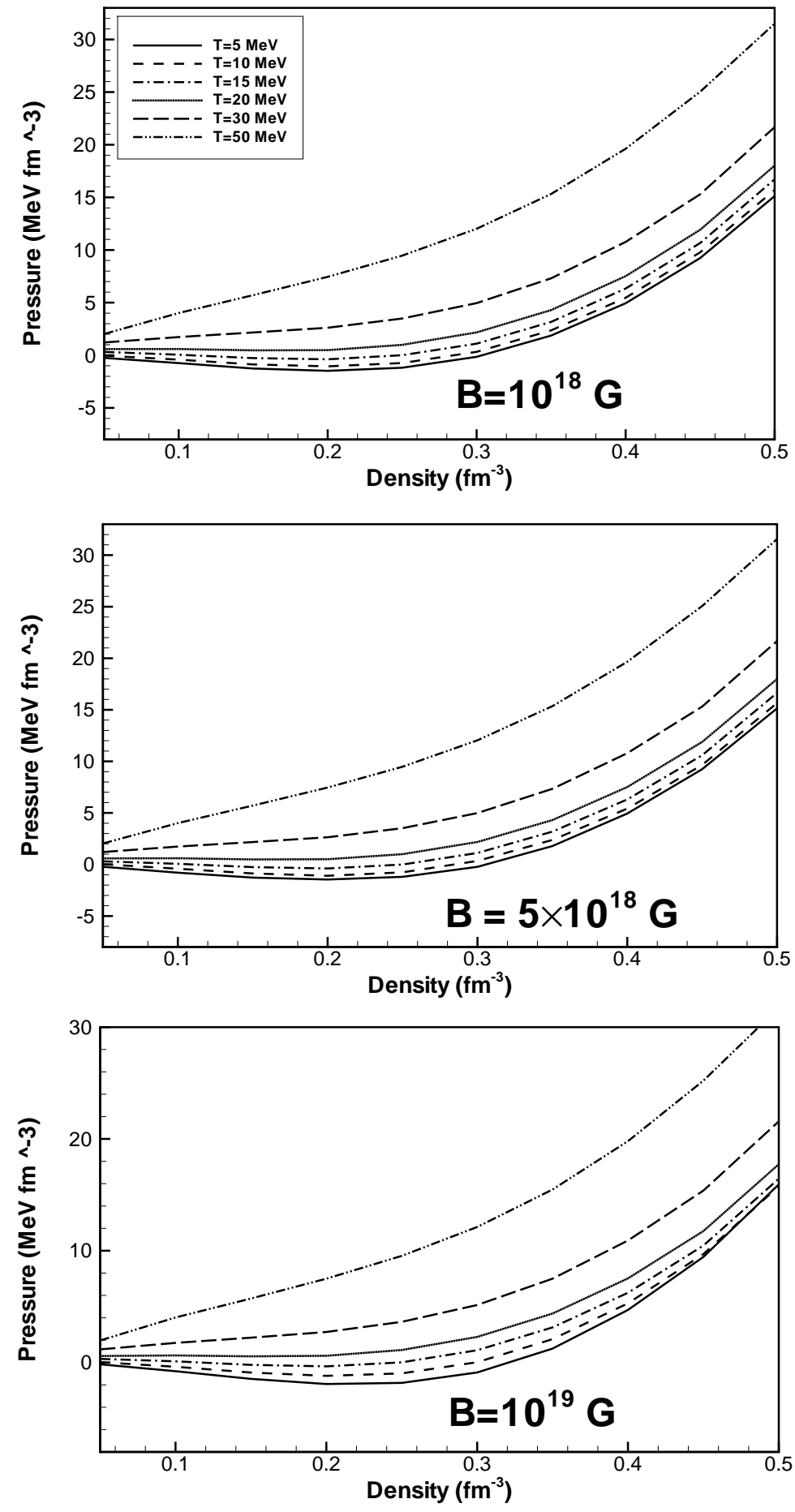

28

FIG. 11: Same as Fig. 10 but at other values of the temperature. 


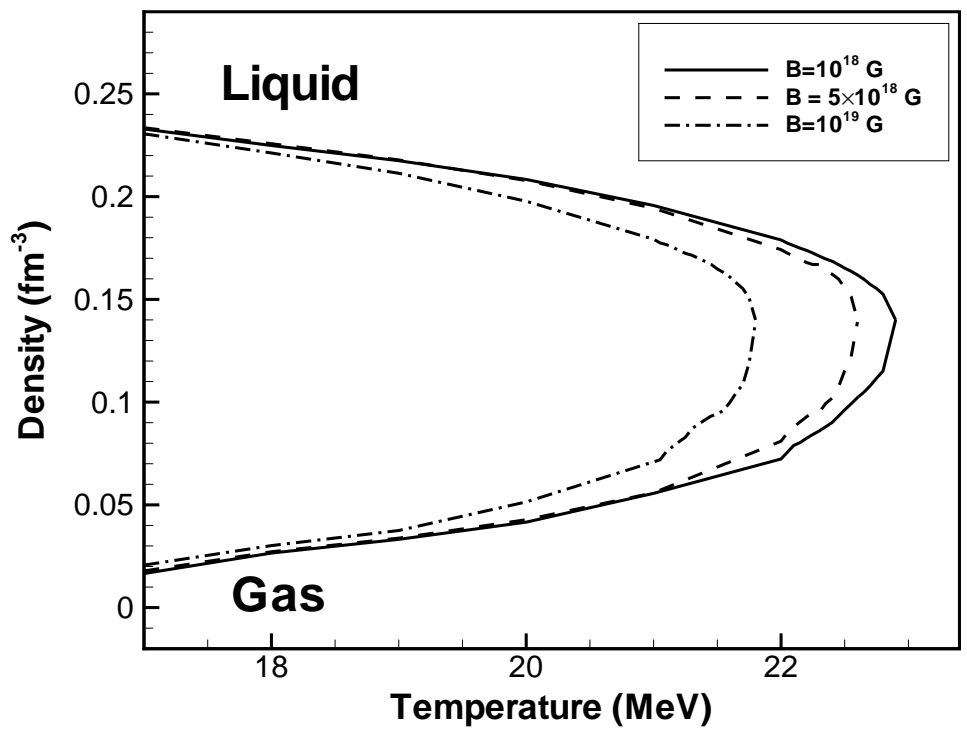

FIG. 12: The liquid gas coexistence curve at different magnetic fields. 


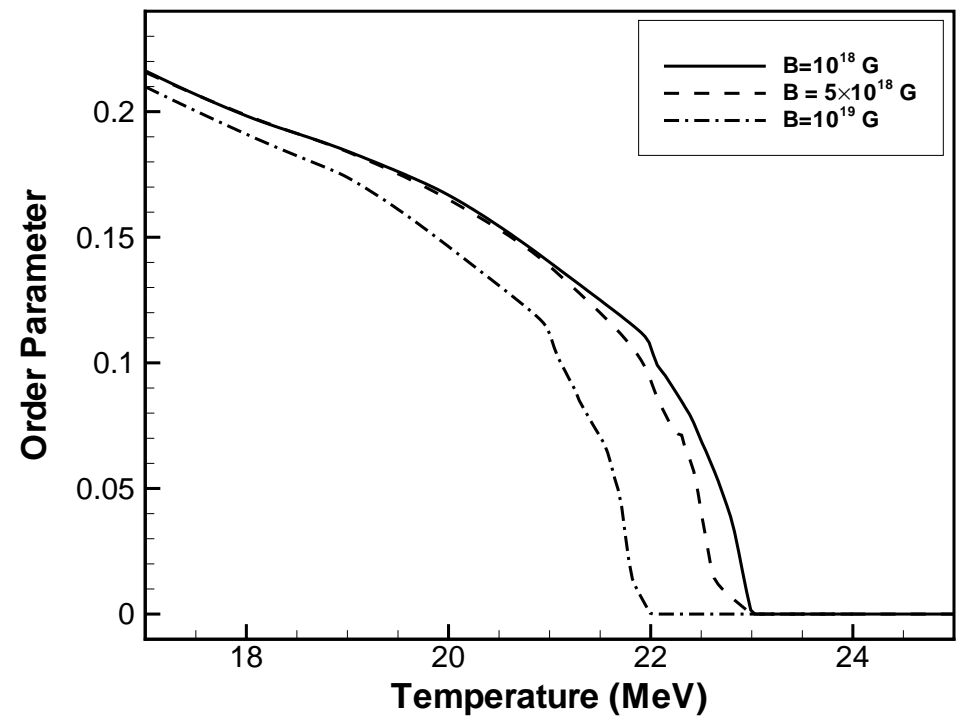

FIG. 13: The order parameter for the liquid gas phase transition as a function of temperature at different magnetic fields. 

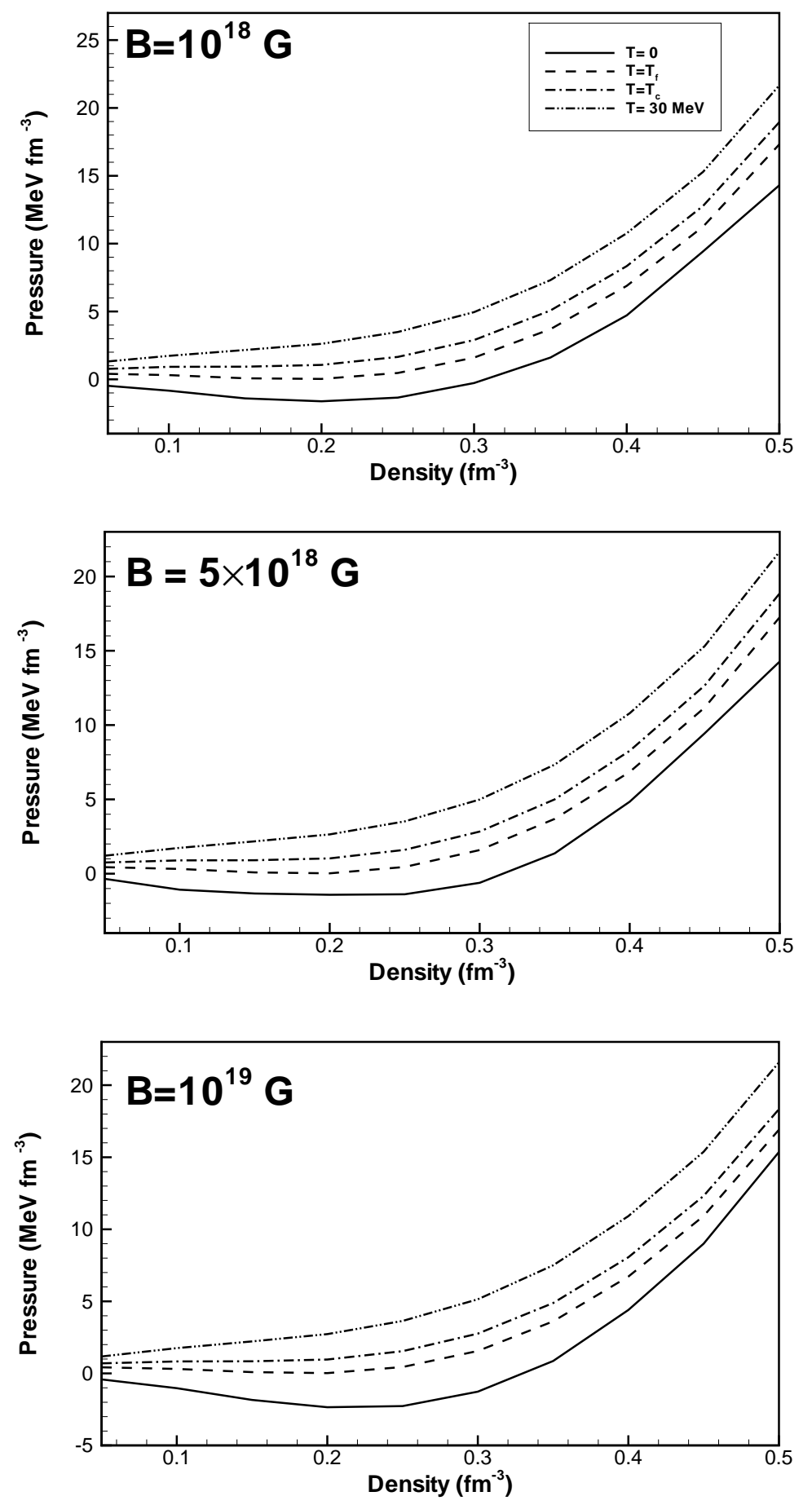